\title{
Prevalence of Heamonchosis in Small Ruminants and Its Associated Risk Factors in and Around Ejere Town, West Shoa, Oromia, Ethiopia
}

\author{
Feyisa Bekuma* and Bayisa Dufera \\ School of Veterinary Medicine, Wollega University, Ethiopia \\ *Corresponding author: Feyisa Bekuma, School of Veterinary Medicine, Collage of Medical and Health Science, Wollega University, \\ P.O. Box 395, Nekemte, Ethiopia.
}

To Cite This Article: Feyisa Bekuma. Prevalence of Heamonchosis in Small Ruminants and Its Associated Risk Factors in and Around Ejere Town, West Shoa, Oromia, Ethiopia. Am J Biomed Sci \& Res. 2019 - 3(5). AJBSR.MS.ID.000704. DOI: 10.34297/AJBSR.2019.03.000704

Received: June 09, 2019 | Published: July 03, 2019

\begin{abstract}
A cross sectional study was conducted from July 2017 to February 2018 for determination of small ruminant haemonchosis and investigation of associated potential risk factors in and around Ejere town, west shoa, Oromia, Ethiopia. The purpose of the study was to determine the prevalence and associated risk factors of Haemonchus in the area. The totals of 384 animals were included in the study from these 261 sheep and 123 goats depending on the number of small ruminants of the district they were taken randomly from whole animals. The overall prevalence was $63.02 \%$ which is lower than the previous reports. This is due the most pastureland is occupied by agricultural activity so that small ruminants are not exposed to this gastrointestinal parasite. The prevalence was higher in sheep (63.6\%) than goats (61.78\%). In case of species there was no statically significant difference $(\mathrm{p}>0.05)$. The prevalence in young was higher as compared with adult small ruminants with prevalence of $74.72 \%$ and $52.47 \%$ in young and adult respectively. Depending on body condition the prevalence was higher in poor body conditioned animals (94.5\%) due to lack of resistant against diseases and lower in good body conditioned animals (30\%). There was significant difference between body condition and Haemonchus prevalence $(\mathrm{p}<0.05)$. from all peasant associations the highest prevalence was recorded in Ilu aga $(75.3 \%)$ and the lowest was observed in Chirri $(48 \%)$ there was no significant difference between origin of animals and haemonchosis ( $p>0.05)$. The prevalence also higher in September $(79.68 \%)$ followed by October (67.96\%) and November (41.4\%). late summer is favorable for Haemonchus to undergo its development and consumed with grass. The prevalence was higher in female (71.6\%) than male (58.4\%) due to females are always under stress of parturition and pregnancy. There is no significant difference between sex and Haemonchus prevalence ( $p>0.05$ ). Further studies on the economic importance of helminthiasis and drug resistance patterns of anthelmintics should be conducted for the holistic implementation of helminthiasis control..
\end{abstract}

Keywords: Haemonchosis; Prevalence; Small Ruminants

\section{Introduction}

Small ruminants are important source of income for agricultural community and are one of Ethiopia's major sources of foreign currency through exportation of live animals, meat and skin [1]. Small ruminants play a great role in the economy of the country, as sources of meat, milk, fiber, cash income and skin and they can live in extreme climatic conditions, they can use herbage, which is unsuitable for large ruminants and they require few labor-intensive inputs [2].

Internal parasites represented an important cause of disease and lose of production in small ruminants [3]. Gastrointestinal nematodes are recognized as a major constraint to both small and large-scale small ruminant production in developing countries [4]. These could be harmful to the health different regions of infected animals and causes economic losses due to mortalities, reduce weight gain and other production losses [5,6].

In topics, the major species of nematodes affecting small ruminants are Heamonchus contortus, Trichostrongylus species, Nematodirus species, Cooperia species, Bunostomum species and Oesophagostomum species. Haemonchus is regarded as one of the most prevalent and highly pathogenic, possesses the highest biotic potential, and has a prominent ability to develop resistance against most widely used anthelmintics and a unique survival strategy due to great biological and ecological plasticity. Hence compared to other gastrointestinal nematodes haemonchus is the most important parasite of domestic ruminants especially in sheep and goats [7-9]. 
H. contortus is blood sucking nematode parasite, primarily occurring in the abomasum of small ruminants, notably sheep and goats. This nematode is also called the barber pole worm because of its red and white striped appearance in the female. The female is capable of producing over 5, 000 eggs a day, which are passed through the feces onto pasture. It has been ranked as the most important parasite of small ruminants in all regions across the tropics and subtropics [10].

Haemonchus contortus is the species of haemonchus with greatest pathogenic and economic importance in small ruminants. It is important to assess the nature and level of parasitism in ruminant livestock, in order to be able to determine significance of parasite infection and to recommend the most beneficial and economically acceptable control measures. The determination of the risk factors associated with parasite occurrence can be used to design an effective control strategy [11]. Major impacts of $\mathrm{H}$. contortus are associated with blood sucking activity of the parasites which responsible for extensive loss of blood [12].

H. contortus causes retarded growth, low productivity, loss of appetite, decrease in protein, impaired digestive efficiency and poor reproductive performance which can lead to loss of meat and wool production $[13,14]$. On global basis $\mathrm{H}$. controtus probably causes more losses than any other species of nematode in ruminants.

Previously there was few documented data regarding to the prevalence of the haemonchosis in small ruminants regardless of the high populations of sheep and goats in the study area. Even though numerous epidemiological studies have been conducted throughout the world to arrive at the detailed information on the gastrointestinal parasites of livestock but there are scanty references are available on epidemiology and prevalence of intestinal helminthes of sheep and goats. According to [11],

Therefore, the objectives of this study were:

a. To determine the prevalence of ovine and caprine Haemonchosis and animal related risk factors in and around Ejere Woreda, Oromia, Ethiopia.

\section{Materials and Method}

\section{Study Area}

The study was conducted in and around Ejere town, including Ejere vet clinic, which is found in western shoa zone, Oromia, Ethiopia. Ejere is approximately $44 \mathrm{~km}$ from Addis Ababa. The elevation is about 2300 to 3000 meters above sea level. The mean annual temperature and relative humidity are 28 and 55\% respectively. An annual rainfall is approximately $1200 \mathrm{~mm}$, with a bimodal distribution pattern peaking in mid-April and mid-August. There are four seasons such as a short rainy season (from midMarch to mid may), a short dry season (from end may to June), long wet season July to October) and a long dry season (end of October to February). Main pasture production is expected after the short rainy season, continuing until the end of the long-wet season. Mixed type of agriculture is the main occupation of the population of the area, a total livestock population of this district was 270789; which
102937 cattle, 12778 goats, 49099 sheep, 20721 donkeys, 165 mules, 12031 horses and 73058 poultry [15].

\section{Study population}

The study animals are ovine and caprine species in study district. 384 small ruminants were taken as a sample in which 261 sheep (81 females and 180 males) and 123 goats (53 females and 70 males). The study animals were indigenous breeds kept under traditional management system with different sex and body condition. Those animals were randomly selected from seven peasant associations of the Woreda; like Ilu Aga, Baso, Damotu, Chiri, Gaba Jimata, Gulit and Ejere 01 kebele. The number of sheep included in the study is large because of high number of sheep exist in the woreda.

\section{Study design and period}

A cross sectional (observational) study was conducted from July 2017 to February 2018 to determine(estimate) the overall prevalence of small ruminants (Ovine and Caprine) Haemonchosis in and around Ejere Woreda. The prevalence was determined in respect to the number of risk factors such as host factors (age, sex, species and body condition of the animals), and the environmental factors included (origin of the animal and months).

\section{Sample Collection and Examination}

The animals were selected by simple random sampling technique. Fecal sample was collected directly from the rectum of selected animal and stored in vials containing 10\% formalin and transported for laboratory examination to Ejere woreda veterinary clinic. The collected samples were undergone flotation technique and examined under microscope for detection of Haemonchus larvae [12]. Randomly selected shoat those come to the district's clinic was used for further detection of the presence of adult parasites and their pathological effects inside the gastrointestinal tract and other body part of the shoat.

\section{Sample Size Determination}

By using simple random sampling methods and $95 \%$ confidence interval with required $5 \%$ precision, the sample size was determined by the formula of [16]. To calculate the total sample size, the following confidence level (CL), 5\% desired level of precision; and parameters will be used: $95 \%$ of with the assumption of $50 \%$ expected prevalence of haemonchosis among sheep and goats in the study area.

$$
\mathrm{n}=\frac{1.96^{2} * \operatorname{Pexp}(1-\operatorname{Pexp})}{\mathrm{d}^{2}}
$$

When: $n=$ required sample size; Pexp= expected prevalence $d=$ desired absolute precision. Hence, by using this formula, the sample size will be calculated to be 384 . Simple random sampling technique was employed to estimate the prevalence of haemonchosis in small ruminant.

\section{Data Analysis}

The data, which was collected from the study area, result obtained from fecal examination was recorded in the format 
developed for this purpose and was entered in to Microsoft Excel spread sheet. Statistical Package for Social Sciences (SPSS, version 20) was used for analysis of the data. The significant association between the prevalence of Haemonchosis and explanatory variables were determined using Chi-square test (x2). The explanatory

\section{Results}

variables included were sex, age, body condition, breed and their associations with the level of prevalence was described. The difference was regarded as significant if P-value is $<0.05$ at $95 \%$ confidence interval.

Table 1: Relative prevalence of haemonchosis in sheep and goats.

\begin{tabular}{|c|c|c|c|c|c|}
\hline Species & No of examined animals & Positive & Prevalence (\%) & $\mathbf{x}^{\mathbf{2}}$ & P-value \\
\hline Sheep & 261 & 166 & 63.6 & 0.118 & 0.408 \\
\hline Goat & 123 & 76 & 61.78 & \\
\hline Total & 384 & 242 & 62.69 & \\
\hline
\end{tabular}

A cross sectional study was carried out to determine the prevalence of haemonchosis in small ruminants from July 2017 to February 2018 in and around Ejere town. In this study a total of 384 sheep and goats were examined using parasitological examination for the presence or absence of $\mathrm{H}$. contortus. The overall prevalence of haemonchosis in small ruminants was found $63.02 \%$ (242) in the study area (Table 1). The prevalence of haemonchosis was found higher in sheep (63.6\%) compare to goats $(61.78 \%)$. There was no statistically significant difference $(P>0.05)$ on the occurrence of haemonchosis between species of animals.

In the present study, higher prevalence of haemonchosis infestation was observed in young animals (74.72\%) as compared to adult $(52.47 \%)$. There was statistically significant difference (P $<0.05$ ) between the two ages groups (Table 2).

Table 2: Relative prevalence of haemonchosis in adult and young age group.

\begin{tabular}{|c|c|c|c|c|c|c|}
\hline Species & Negative & Positive & Total & Prevalence (\%) & $\mathbf{X}^{2}$ test & P-value \\
\hline Adult & 96 & 106 & 202 & 52.47 & 20.338 & 0 \\
\hline Young & 46 & 136 & 182 & 74.72 & & \\
\hline Total & 142 & 242 & 384 & & & \\
\hline
\end{tabular}

The prevalence of haemonchosis was different with different body condition of small ruminants. It was found slightly higher in poor $(94.5 \%)$ then medium body condition with prevalence of $(63.1 \%)$ and followed by good body condition (30\%). There was

significant difference (P number of sheep and goats harboring the parasite by the $<0.05$ ) between body conditions of small ruminants (Table 3).

\begin{tabular}{|c|c|c|c|c|c|}
\hline \multicolumn{2}{|l|}{ Table 3: prevalence of haemonchosis based on body condition score. } \\
\hline Body condition & Negative & Positive & Prevalence (\%) & $\mathbf{X}^{2}$ & p-value \\
\hline Good & 79 & 34 & 30 & & 0 \\
\hline Medium & 56 & 96 & 63.1 & & \\
\hline Poor & 7 & 122 & 94.5 & & \\
\hline Total & 142 & 242 & & \\
\hline
\end{tabular}

Table 4: Prevalence of haemonchosis in peasant associations.

\begin{tabular}{|c|c|c|c|c|c|}
\hline Peasant associations & Negative & Positive & Prevalence (\%) & $\mathbf{X}^{2}$ test & p-value \\
\hline Chirri & 27 & 25 & 48 & 61.303 & 0.79 \\
\hline Ejere 01 & 18 & 29 & 55.5 & & \\
\hline Dhamotu & 24 & 30 & 66.6 & & \\
\hline Baso & 18 & 36 & 64 & & \\
\hline Gulit & 18 & 32 & 65.5 & & \\
\hline G/ jimata & 20 & 38 & 75.3 & & \\
\hline Ilu aga & 17 & 242 & 63 & & \\
\hline Total & 142 & & & \\
\hline
\end{tabular}

The prevalence of haemonchosis was also different among peasant associations of the study area. The highest prevalence of haemonchosis was observed in Ilu Aga (75.3\%) and the lowest prevalence was recorded in Chirri peasant associations (48\%). There was no significant difference among peasant association and haemonchus in study area (Table 4). 
Table 5: The prevalence of haemonchosis in both sexes of the animals.

\begin{tabular}{|c|c|c|c|c|c|}
\hline Sex of animal & Negative & Positive & Prevalence (\%) & $\mathbf{X}^{2}$ test & p-value \\
\hline Male & 104 & 146 & 58.4 & & 0.564 \\
\hline Female & 38 & 96 & 71.6 & & \\
\hline Total & 142 & 242 & 67.5 & \\
\hline
\end{tabular}

The prevalence of small ruminant haemonchosis was different between both sexes. It was found higher in females (71.6\%) than male $(58.4 \%)$ in the study area. There was no significant difference between sexes of small ruminants (Table 5).

\section{Discussion}

The present study revealed an overall prevalence of haemonchosis infestation in small ruminants was found to be $63 \%$. The prevalence among species was $63.6 \%$ in sheep and $61.78 \%$ in goats of the area which indicate there is still a high prevalence of haemonchosis in the small ruminants. This finding is lower than the results of previous works in sheep and goats from different parts of Ethiopia [17] reports $90.1 \%$ in sheep and $81.8 \%$ in goats in the prevalence of abomasal nematodes in sheep and goats slaughtered at $[17,18]$ report $96.5 \%$ in sheep and $100 \%$ in goats in the arid and semi-arid zone of eastern Ethiopia; [19] report $91.2 \%$ in sheep and $82.9 \%$ in goats of Ogaden region slaughtered at Debre-zeit (Bishoftu) ELFORA export abattoir; [20] in the study conducted in and around Finoteselam has reported prevalence of haemonochosis among small ruminants as $71.03 \%$ and [21] also reported higher prevalence in the study of an Abattoir Survey on Gastrointestinal Nematodes in Sheep and Goats in Helmex-Export Abattoir as $77.38 \%$.

This deference could be due to difference in agro-climatic conditions that could support extended survival and development of infective larval stage of $\mathrm{H}$. contortus as well as most pastureland is occupied by agricultural practices. Moreover, this difference might be due to the difference in management system of examined animals, sample size and environmental location of the area. And also, might be due to small ruminants are managed under extensive managements system with the high stocking density, where large numbers of animals graze together throughout the year in collective grazing land and insufficient nutritional status [22].

A variety of factors such as, age, host, level of education and economical capacity of the community, the standard of management and anthelmintics usage, presence or absence of inter- current infections are critical elements influencing the development, distribution and survival of parasites [23]. Other factors that provoke this variation might be due to frequency of adequate rainfall and wetness during the study period was favored the survival of infective larvae in grazing land and higher chance of uptake of the infective larvae that basis for higher prevalence. The percentage prevalence of $\mathrm{H}$. contortus in sheep and goats was recorded as $63.6 \%$ and $61.78 \%$ respectively. There was no significant difference $(\mathrm{P}>0.05)$ in the prevalence of haemonchosis between sheep and goats, indicating that sheep's are more susceptible to the infection than goats.
The results of the present study are supported by [22] who reported $81.2 \%$ and $73.5 \%$ in sheep and goats respectively in Gonder town; [24], who reported $69.5 \%$ and $65 \%$ in sheep and goats in and around Bedelle, [25] who reported 61.63\% and $54.76 \%$ in sheep and goats respectively in and around Wolaita Soddo. However, the current study is disagreeing with [20]. Who reported that the rate of the parasite was higher in goat compared to sheep with the prevalence of $71.3 \%$ and $67.57 \%$ respectively? The higher prevalence of haemonchosis in sheep than goats also might be due to a diversity of factors like ground grazing habit of sheep and usually graze very close to the soil which might be helpful in the acquisition of more infective larvae (L3) of $\mathrm{H}$. contortus from the contaminated herbage. Additionally, high prevalence of haemonchosis in sheep than goat might be due to the fact that goats browse on bushes and small trees where translation of infective larvae to such height seems impossible.

The present study revealed that there was significant difference based on age with prevalence of $74.72 \%$ and 52.47 in young and adults respectively. The present finding on the prevalence of haemochosis between two ages was in line with previous findings which were reported by [21] as $86.9 \%$ and $86.57 \%$ in adult and young respectively in Helmex-export abattoir and [26] who reported $37.9 \%$ and $49 \%$ in young and adult respectively in and around Alameta Woreda. This might be due to that; the more infection in young is because of their low resistance or greater susceptibility due to the fact that these small ruminants have not been exposed earlier in the infection.

During the first year of their life they fed, grazed and browse on grasslands, thus the first stage of their exposure to infection with parasites occurs. It was also explained that low level of parasitism reported in the adult animals is due to the development of significant immunity with the course of time. Gradually, as the exposure to parasitic infection increases, the immune system of host animals builds up to against Haemonchus spp and age resistance develops [27].

With regard to the body condition of the examined small ruminants the prevalence rate was higher in poor body condition small ruminants as compared to the good and medium body conditions small ruminants with the prevalence of $94.5 \%$, in poor body conditioned shoat, $63.1 \%$ in medium body condition and $61.5 \%$, in good body condition small ruminants. There was statistically significant $(\mathrm{P}<0.05)$ between body condition and haemonchosis in small ruminants this indicated that all the three body condition animals were unequally susceptible for haemonchosis. The prevalence of haemonchus was high in poor body conditioned small ruminants this could be explained by 
poor body condition animals susceptible to parasitic diseases and they cannot resist against diseases due to their immunity become lowered agree with [28] who reported the highest prevalence was seen in poor body conditioned animals (33.6\%) while the lowest in good of revealed that body condition.

This current study was agree with the previous works of [21] who reported prevalence of $77.21 \%$ and $84.44 \%$ in good and medium body condition animal; [29] indicated that the rate of the parasite was higher in medium body condition small ruminants compared to that of good body condition with the prevalence of 67.3 and 55\% respectively in small Ruminants Haemonchosis.

Prevalence and Associated Risk Factors in Arsi Negelle Municipal Abattoir, Similarly, [22] indicated that the rate of the parasite was higher in medium body condition small ruminants than that of good body condition small ruminants with the prevalence of $81.2 \%$ and $73.6 \%$, respectively; but disagrees with the research reported by [30] who report prevalence of haemonchosis was found to be higher in good body condition than that medium body condition. This could be explained by the fact that loss of body condition in the study animals might be due to other factors, such as seasonal change of forgeable feed staff, Poor management system and the presence of other concurrent diseases which lead to poor immunological response to infective stage of the parasites [31].

The present study revealed that the prevalence of haemonchus was higher in female than male with prevalence of $71.6 \%$ and $58.4 \%$ in female and male respectively. There was no significant difference between prevalence of haemonchus and sex of the animal it means that both sexes are equally susceptible for haemonchus. The present finding on the prevalence of haemonchosis between sexes was disagree with previous findings which were reported by [22] as $80.9 \%$ and $77.1 \%$ in males and females respectively in Gonder town and [20] who reported $73.22 \%$ and $64.71 \%$ in male and females respectively in and around Finoteselam in Amhara region. It is assumed that sex is a determinant factor influencing prevalence of haemonchosis and females are more susceptible to parasitism during pregnancy and Per-parturient period due to stress and decreased immune status.

The present study forwarded that the prevalence of haemonchus was different among animals originated from different sites of study areas. The highest prevalence was observed on animals originated from Ilu aga (75.3\%), Baso (66.6\%), Gaba Jimata (65.5\%), Gulit (64\%) and the lowest prevalence observed in Ejere 01 (61\%), Dhamotu (55.5\%), and Chirri (48\%). There is no statically significant difference between peasant association and prevalence of haemonchosis. This is might be due to the fact that sheep and goats are managed under extensive management system with high stocking density graze together throughout the year in communal designed grazing land which leads to more contamination of a pasture. Another possible explanation might be sheep and goat graze in a rotation with cattle is due to inadequate nutritional status, poor pasture. The difference in prevalence of parasite problems in different location might be due to the difference between the geographical and environmental location of the area.

\section{Conclusion and Recommendations}

Small ruminants are an integral part of the livestock sector and the mainstay of livelihood of the majority population especially in developing countries like Ethiopia. However, the economic benefits remain marginal chiefly due to prevailing disease. Among the diseases of sheep and goats that reduce the survival and productivity of small ruminants is Haemonchus contortus being of over wheeling significance. It is blood sucking parasite and causes loss of production, weight loss and death. In present study the overall prevalence of haemonchosis in small ruminants was found to be $63 \%$. The prevalence among species was $63.6 \%$ in sheep and $61.78 \%$ in goats, even though this finding is lower than the results of previous works in sheep and goats from different parts of Ethiopia it still indicates a high prevalence. The higher prevalence of Haemonchus contortus in this study area is mostly associated with the epidemiological factors such as body condition and age were statistically significant whereas, species of animal, sex and origin of the animals were statistically not significant. The epidemiological evidence of the present investigation showed that Haemonchosis is considerably prevalent diseases in the study area. Generally, the presence of risk factors like warm and humidity, poor animal management, and seasonal scarcity of feed and other concurrent diseases in the study area favors the disease of distribution.

Based on the above conclusion the following recommendations are forwarded:

a. There should be rotational grazing of pastureland to control Haemonchus parasites

b. Regular deworming should be implemented to reduce the population of the parasites in the animals.

c. Further studies on the economic importance of haemonchosis and drug resistance patterns of anthelmintics should be conducted.

d. Improving the management of the small ruminant by considering the period of peak haemonchosis prevalence.

\section{Competing Interests}

All authors have declared that no competing interests exist.

\section{Acknowledgements}

The authors would like to thank Wollega University, School of Veterinary Medicine for financial and logistics, study participants and all individuals who render help during the study period.

\section{Author Contributions}

Contributed to designing the methodology and collecting materials: Bayisa Dufera, correcting of the methodology and checking up for collected materials: Bayisa Dufera and Feyisa Bekuma, contributed to writing the manuscript: Feyisa Bekuma: Reviewing the manuscript to be ready for publication and contributes on drafting of the manuscript.

\section{References}

1. Sheferaw D, H Degefu, D Banteyirgu (2010) Epidemiological study of small ruminant mange mites in three agro-ecological zones of Wolaita, Southern Ethiopia. Ethio Vet J 14: 31-38. 
2. Fraser CM (1991) A Handbook of Diagnosis, The Merck burden and species composition. Rev Med Vet Veterinary ( $7^{\text {th }}$ edn)

3. Zajac AM (2006) Gastrointestinal nematodes of small ruminants: Life cycle, anthelminthes and diagnosis.Vet Clin N AM Food Anim Practice 22(3): 529-541.

4. Martinez Gonzalez B, Diez Banos N, Rojo Vaz Quuez FA (1998) An epidemiological Study of Gastrointestinal Parasitism in dairy sheep flocks in Leon (NW Spain). Small Rumin Res 27(1): 25-30.

5. Skerman KO, Shahlapoor AA, Eslami A, Eliazian M (1970) Observation on the incidence, epidemiology, control and economic importance of gastrointestinal parasites of Sheep and Goats in Iran. Veterinary Medical Review 22: 187-196.

6. Sissay M, Uggla A, Waller P (2007) Epidemiology and seasonal dynamics of Gastrointestinal nematode infections of sheep in a semi-arid region of eastern Ethiopia, Veterinary Parasitol 143(3-4): 311-321.

7. Bekele T, Woldeab T, Lahlou Kassi A, Sherngton J (1992) Factors Affecting Morbidity and Mortality on Farm and On Station in Ethiopia Highland Sheep. Acta Trop 52(2-3): 99-109.

8. Le Jambre LF (1995) Relationship of blood loss to worm numbers, biomass and egg production in haemonchus infected sheep. Int J Parasitol 25(3): 269-273.

9. Perry B, Randolph T, Mcdermott S, Ones K, Tornton PK (2002) Investing in Animal Health Research to alleviate Poverty, (International Livestock Research Institute (ILRI), Nairobi, Kenya, East Africa, pp. 148.

10. Bhat SA, Mir MR, Allaie SQ, Khan HM, Husain I, et al. (2011) Comparative Resistance of Sheep Breeds to Haemonchus Contortus in Pasture Infection in Jammu and Kashmir. Global Veterinaria 8: 222-228.

11. Odoi A, JM Gathuma, CK Gachuiri, A Omore (2007) Risk factors of gastrointestinal nematode parasite infections in small ruminants kept insmallholder mixed farms in Kenya. BMC Veterinary Research 3(6): 59 70.

12. Urquhart GM, Armour J, Duncan JL, Dunn AM, Jennings FW (2003) Veterinary parasitology, 2. Ed. Scotland, balack well Science, pp: 276228.

13. Iqbal Z, A Jabbar (2005) Dealing with a threat.

14. Bachaya HA, Z Iqbal, A Jabbar, R Ali (2006) Copping with loss of livestock.

15. Agricultural sample survey (2011) Vol. II. Report on livestock and livestock characteristics. Statistical Bulletin, Addis Ababa, Ethiopia, CSA

16. Thrusfield M (2018) John Wiley \& Sons Ltd, Veterinary epidemiology. ( $4^{\text {th }}$ edn), pp. 233.

17. Argaw S, Desta B, Belay A (2014) Prevalence of abomasal nematodes in sheep and goats slaughtered at Haramaya municipal abattoir, eastern Hararghe, Ethiopia. J Bio Agr Heal 4(26): 2224-3208.
18. Wossene A, Gelaye E (2001) Survey of ovine and caprine gastrointestinal helminthiasis parts of Ethiopia during the dry season of the year. Rev de Med Vet 152(5): 379-384.

19. Kumsa B, Wossene A (2006) Abomasal nematodes of small ruminant of Ogaden region Eastern Ethiopia: prevalence, worm burden and species composition. Rev Vet Med 157(12): 27-32.

20. Mengist Z, Abebe N, Gugsa G, Kumar N (2014) Assessment of Small Ruminant Haemonchosis and Its Associated Risk Factors in and Around Finoteselam, Mekelle University, College of Veterinary Medicine, Ethiopia. J Agr Vet Scie 4: 36-41.

21. Shankute G, Basaznew B, Achenef M (2013) An abattoir survey on gastrointestinal nematodes in sheep and goats in hemex export. J Adv Vet Resea 3: 60-63.

22. Tewodros F, Girja L (2012) Small Ruminant Haemonchosis: Prevalence and Associated Determinants in Randomly Selected Restaurants and Hotels of Gondar Town, Ethiopia. Eur J Appl Scie 4(4): 168-172.

23. Rahmeto A, Meberahtu G, Solomon R, Fufa A, Alemayehu R (2010) Gastrointestinal nematode infection in small ruminants under the traditional husbandry systems during the dry season in Southern Ethiopia. Trop Ani Heal 42(6): 1111-1117

24. Mulugeta T, Batu G, Bitew B (2011) Prevalence of Gastrointestinal Parasites of Sheep and Goats in and and Around Bedelle, South-Western Ethiopia. Inter J Vet Med 8: 134-156.

25. Haileleul N (2002) Prevalence of gastrointestinal helminthes of small ruminants in and Around Wolayitasodo, Southern Ethiopia. p. 1-35.

26. Meselea H, K/yohannesa T, Zegeye S (2013) Prevalence of haemonchosis in small ruminants in and around Woreda Alameta. Rev Electr Vet 14(4): $1-12$.

27. Seth B (2014) A Review of the Epidemiology of Gastrointestinal Nematode Infections in Sheep and Goats in Ghana. J Agri Scie 6(4): 19169760 .

28. Gonfa Shankute, Basaznew Bogale, Achenef Melaku, MS Akhtar (2005) An Abattoir Survey on Gastrointestinal nematode in Sheep and Goats in Hemex-Export, Debre Ziet, Central Ethiopia. Journal of Advanced Veterinary Research 3(2): 60-63.

29. Tibeso B, Mekonnen M (2015) Small Ruminants Haemonchosis: Prevalence and Associated Risk Factors in ArsiNegelle Municipal Abattoir, Ethiopia. Glob Vet 15(3): 315-320.

30. Regassa A, Toyeb M, Abebe R, Megersa B (2010) parts of Ethiopia during the dry season of the year. B. Mekibib, et al. (ED's) Prevalence and associated risk factors in Dessie and Kombolcha districts, northeastern Ethiopia. Vet Parasitol 169: 144-148.

31. Southcoatt WH, Hajor GW, Barger IA (1996) Seasonal pasture contamination and availability of nematodes for grazing sheep. Australian Journal of Agricultural Research 27(2): 277-286. 